\title{
Carbonaceous aerosol composition in air masses influenced by large- scale biomass burning: a case-study in Northwestern Vietnam
}

Dac-Loc Nguyen ${ }^{1,2,8}$, Hendryk Czech ${ }^{1,2}$, Simone M. Pieber ${ }^{3}$, Jürgen Schnelle-Kreis ${ }^{1}$, Martin Steinbacher $^{3}$, Jürgen Orasche ${ }^{1}$, Stephan Henne ${ }^{3}$, Olga B. Popovicheva ${ }^{4}$, Gülcin Abbaszade ${ }^{1}$, Günter Engling ${ }^{5, \mathrm{a}}$, Nicolas Bukowiecki ${ }^{6}$, Nhat-Anh Nguyen ${ }^{7}$, Xuan-Anh Nguyen ${ }^{8}$, Ralf Zimmermann ${ }^{1,2}$

${ }^{1}$ Joint Mass Spectrometry Centre (JMSC), Cooperation Group "Comprehensive Molecular Analytics" (CMA), Helmholtz Zentrum München, München, 81379, Germany

${ }^{2}$ Joint Mass Spectrometry Centre (JMSC), Chair of Analytical Chemistry, University of Rostock, Rostock, 18059, Germany

${ }^{3}$ Empa, Laboratory for Air Pollution/Environmental Technology, Dübendorf, Switzerland

${ }^{4}$ Skobeltsyn Institute of Nuclear Physics, Moscow State University, Moscow, 119991, Russian Federation

${ }^{5}$ Department of Biomedical Engineering and Environmental Sciences, National Tsing Hua University, Hsinchu 30013, Taiwan

${ }^{6}$ Department of Environmental Sciences, University of Basel, Basel, 4056, Switzerland

${ }^{7}$ Hydro-Meteorological Observation Center, Vietnam Meteorological and Hydrological Administration, Ministry of Natural Resources and Environment, Ha Noi, Vietnam

${ }^{8}$ Institute of Geophysics, Vietnam Academy of Science and Technology (VAST), Ha Noi, Vietnam

anow at: Mobile Source Laboratory Division, California Air Resource Board, El Monte, CA 91731, United States

Correspondence to: Hendryk Czech (hendryk.czech@uni-rostock.de; hendryk.czech@helmholtz-muenchen.de) 
Table S1. EC fractions, OC fractions and speciated organic compounds in $\mathrm{PM}_{2.5}$ samples, including minimum (min.) and maximum (max.), as well as median, mean, $1^{\text {st }}$ and $3^{\text {rd }}$ quartiles (Q1 and Q3).

\begin{tabular}{|c|c|c|c|c|c|c|c|c|}
\hline & Abbreviation & Unit & Min. & Q1 & Median & Q3 & Max. & Mean \\
\hline Organic Carbon fraction & $\mathrm{OC} 1$ & $\mu \mathrm{g} \mathrm{m}^{-3}$ & 0.00 & 0.00 & 0.12 & 0.42 & 1.05 & 0.23 \\
\hline Organic Carbon fraction & $\mathrm{OC} 2$ & $\mu \mathrm{g} \mathrm{m}^{-3}$ & 0.38 & 0.64 & 1.21 & 1.87 & 7.07 & 1.73 \\
\hline Organic Carbon fraction & OC3 & $\mu \mathrm{g} \mathrm{m}^{-3}$ & 1.21 & 2.38 & 4.11 & 6.94 & 15.30 & 5.23 \\
\hline Organic Carbon fraction & $\mathrm{OC} 4$ & $\mu \mathrm{g} \mathrm{m}^{-3}$ & 0.21 & 0.74 & 1.58 & 3.05 & 7.96 & 2.25 \\
\hline Pyrolyzed Organic Carbon & OP & $\mu \mathrm{g} \mathrm{m}^{-3}$ & 0.00 & 0.03 & 0.76 & 2.89 & 8.29 & 1.85 \\
\hline Corrected Element Carbon & EC1-OP & $\mu \mathrm{g} \mathrm{m}^{-3}$ & 0.07 & 0.62 & 1.34 & 3.60 & 15.31 & 2.99 \\
\hline Elemental Carbon fraction & $\mathrm{EC} 2$ & $\mu \mathrm{g} \mathrm{m}^{-3}$ & 0.06 & 0.48 & 1.22 & 2.05 & 3.09 & 1.25 \\
\hline Elemental Carbon fraction & EC3 & $\mu \mathrm{g} \mathrm{m}^{-3}$ & 0.00 & 0.00 & 0.04 & 0.12 & 0.43 & 0.10 \\
\hline Organic Carbon & $\mathrm{OC}$ & $\mu \mathrm{g} \mathrm{m}^{-3}$ & 1.82 & 3.73 & 6.57 & 16.0 & 38.33 & 11.1 \\
\hline Elemental Carbon & $\mathrm{EC}$ & $\mu \mathrm{g} \mathrm{m}^{-3}$ & 0.13 & 0.83 & 2.05 & 2.98 & 9.80 & 2.41 \\
\hline Eicosanoic acid & $\mathrm{A}-\mathrm{C} 20$ & $n g m^{-3}$ & 0.03 & 0.04 & 0.08 & 0.23 & 0.66 & 0.17 \\
\hline Docosanoic acid & $\mathrm{A}-\mathrm{C} 21$ & $n g \mathrm{~m}^{-3}$ & 0.06 & 0.15 & 0.43 & 1.53 & 5.85 & 1.14 \\
\hline Tetracosanoic acid & $\mathrm{A}-\mathrm{C} 24$ & $\mathrm{ng} \mathrm{m}^{-3}$ & 0.05 & 0.23 & 0.76 & 3.00 & 12.46 & 2.25 \\
\hline Pentacosanoic acid & $\mathrm{A}-\mathrm{C} 25$ & $n g \mathrm{~m}^{-3}$ & 0.01 & 0.03 & 0.16 & 0.69 & 3.17 & 0.53 \\
\hline Hexacosanoic acid & A-C26 & $n g \mathrm{~m}^{-3}$ & 0.03 & 0.12 & 0.62 & 2.30 & 11.38 & 1.86 \\
\hline Heptacosanoic acid & $\mathrm{A}-\mathrm{C} 27$ & $n g \mathrm{~m}^{-3}$ & 0.00 & 0.01 & 0.10 & 0.44 & 2.25 & 0.36 \\
\hline Octacosanoic acid & $\mathrm{A}-\mathrm{C} 28$ & $n g \mathrm{~m}^{-3}$ & 0.00 & 0.06 & 0.46 & 1.87 & 9.43 & 1.55 \\
\hline Nonacosanoic acid & A-C29 & $\mathrm{ng} \mathrm{m}^{-3}$ & 0.00 & 0.02 & 0.04 & 0.37 & 1.85 & 0.30 \\
\hline Triacontanoic acid & $\mathrm{A}-\mathrm{C} 30$ & $\mathrm{ng} \mathrm{m}^{-3}$ & 0.00 & 0.02 & 0.26 & 1.34 & 6.58 & 1.10 \\
\hline Hentriacontanoic Acid & A-C31 & $\mathrm{ng} \mathrm{m}^{-3}$ & 0.00 & 0.00 & 0.03 & 0.12 & 0.92 & 0.14 \\
\hline Dotriacontanoic Acid & A-C32 & $\mathrm{ng} \mathrm{m}^{-3}$ & 0.00 & 0.00 & 0.13 & 0.67 & 3.16 & 0.52 \\
\hline Galactosan & GAL & $\mathrm{ng} \mathrm{m}^{-3}$ & 0.05 & 0.14 & 0.59 & 1.85 & 8.98 & 1.56 \\
\hline Mannosan & MAN & $\mathrm{ng} \mathrm{m}^{-3}$ & 0.68 & 1.45 & 5.28 & 18.6 & 60.2 & 12.9 \\
\hline Levoglucosan & LEV & $\mathrm{ng} \mathrm{m}^{-3}$ & 23.4 & 110 & 188 & 580 & 1710 & 437 \\
\hline Vanillin & VAH & $\mathrm{ng} \mathrm{m}^{-3}$ & 0.17 & 0.46 & 0.94 & 3.15 & 11.8 & 2.31 \\
\hline p-Hydroxybenzoic acid & p-H-acid & $\mathrm{ng} \mathrm{m}^{-3}$ & 0.31 & 2.12 & 7.60 & 43.0 & 192 & 34.1 \\
\hline m-hydroxybenzoic acid & m-H-acid & $\mathrm{ng} \mathrm{m}^{-3}$ & 0.35 & 1.48 & 4.66 & 16.6 & 45.3 & 10.5 \\
\hline Syringaldehyde & SYAH & $\mathrm{ng} \mathrm{m}^{-3}$ & 0.00 & 0.63 & 1.11 & 6.24 & 40.2 & 6.09 \\
\hline Syringic acid & SYA & $n g \mathrm{~m}^{-3}$ & 0.12 & 0.32 & 1.37 & 7.41 & 47.9 & 7.73 \\
\hline Vanillic acid & VA & $\mathrm{ng} \mathrm{m}^{-3}$ & 0.14 & 0.35 & 2.08 & 9.58 & 49.1 & 9.07 \\
\hline 4-Nitrophenol & $4-\mathrm{NP}$ & $\mathrm{ng} \mathrm{m}^{-3}$ & 0.40 & 0.79 & 2.21 & 5.23 & 59.8 & 6.66 \\
\hline 4-Nitrocatechol & $4-\mathrm{NC}$ & $\mathrm{ng} \mathrm{m}^{-3}$ & 0.20 & 0.45 & 2.32 & 5.19 & 616 & 41.5 \\
\hline 2,6-Dimethoxy-4-nitrophenol & 2,6-D-4-NP & $n g m^{-3}$ & 0.00 & 0.00 & 0.59 & 0.77 & 6.06 & 0.81 \\
\hline
\end{tabular}




\begin{tabular}{|c|c|c|c|c|c|c|c|c|}
\hline Eicosane & $\mathrm{C} 20$ & $\mathrm{ng} \mathrm{m}^{-3}$ & 0.19 & 0.29 & 0.52 & 1.11 & 2.08 & 0.78 \\
\hline Heneicosane & $\mathrm{C} 21$ & $\mathrm{ng} \mathrm{m}^{-3}$ & 0.60 & 0.85 & 1.57 & 2.93 & 4.69 & 1.94 \\
\hline Docosane & $\mathrm{C} 22$ & $\mathrm{ng} \mathrm{m}^{-3}$ & 0.44 & 0.82 & 1.85 & 4.38 & 11.8 & 3.12 \\
\hline Tricosane & $\mathrm{C} 23$ & $\mathrm{ng} \mathrm{m}^{-3}$ & 0.48 & 1.17 & 2.70 & 9.51 & 17.5 & 5.31 \\
\hline Tetracosane & $\mathrm{C} 24$ & $\mathrm{ng} \mathrm{m}^{-3}$ & 0.78 & 2.05 & 4.02 & 7.39 & 16.1 & 5.84 \\
\hline Pentacosane & $\mathrm{C} 25$ & $\mathrm{ng} \mathrm{m}^{-3}$ & 0.45 & 1.13 & 3.01 & 6.14 & 19.5 & 4.82 \\
\hline Hexacosane & $\mathrm{C} 26$ & $\mathrm{ng} \mathrm{m}^{-3}$ & 0.54 & 1.40 & 2.29 & 4.64 & 16.9 & 4.02 \\
\hline Heptacosane & $\mathrm{C} 27$ & $\mathrm{ng} \mathrm{m}^{-3}$ & 0.61 & 1.68 & 3.06 & 10.24 & 31.0 & 7.01 \\
\hline Octacosane & $\mathrm{C} 28$ & $\mathrm{ng} \mathrm{m}^{-3}$ & 0.36 & 1.03 & 1.95 & 4.93 & 17.8 & 4.06 \\
\hline Nonacosane & $\mathrm{C} 29$ & $\mathrm{ng} \mathrm{m}^{-3}$ & 0.53 & 1.49 & 4.55 & 18.46 & 58.8 & 12.7 \\
\hline Triacontane & C30 & $\mathrm{ng} \mathrm{m}^{-3}$ & 0.54 & 0.92 & 1.73 & 4.21 & 11.5 & 3.10 \\
\hline Hentriacontane & $\mathrm{C} 31$ & $\mathrm{ng} \mathrm{m}^{-3}$ & 0.49 & 1.09 & 4.59 & 16.69 & 43.4 & 10.4 \\
\hline Dotriacontane & $\mathrm{C} 32$ & $\mathrm{ng} \mathrm{m}^{-3}$ & 0.17 & 0.36 & 0.82 & 1.89 & 6.27 & 1.48 \\
\hline Tritriacontane & C33 & $\mathrm{ng} \mathrm{m}^{-3}$ & 0.22 & 0.44 & 1.63 & 4.95 & 14.4 & 3.47 \\
\hline Phenanthrene & PHE & $\mathrm{ng} \mathrm{m}^{-3}$ & 0.00 & 0.09 & 0.13 & 0.17 & 0.33 & 0.14 \\
\hline Fluoranthene & FLU & $\mathrm{ng} \mathrm{m}^{-3}$ & 0.01 & 0.03 & 0.03 & 0.05 & 0.09 & 0.04 \\
\hline Pyrene & PYR & $\mathrm{ng} \mathrm{m}^{-3}$ & 0.01 & 0.02 & 0.03 & 0.05 & 0.13 & 0.04 \\
\hline Benz[a]anthracene & $\mathrm{BaA}$ & $\mathrm{ng} \mathrm{m}^{-3}$ & 0.01 & 0.01 & 0.02 & 0.04 & 0.18 & 0.04 \\
\hline Chrysene & CHR & $\mathrm{ng} \mathrm{m}^{-3}$ & 0.02 & 0.03 & 0.03 & 0.06 & 0.17 & 0.05 \\
\hline$\sum$ Benzo[b,k]fluoranthene & $\mathrm{BbkF}$ & $\mathrm{ng} \mathrm{m}^{-3}$ & 0.04 & 0.10 & 0.16 & 0.28 & 0.67 & 0.23 \\
\hline Benz[e]pyrene & $\mathrm{BeP}$ & $\mathrm{ng} \mathrm{m}^{-3}$ & 0.03 & 0.09 & 0.16 & 0.25 & 0.42 & 0.19 \\
\hline Benz[a]pyrene & $\mathrm{BaP}$ & $\mathrm{ng} \mathrm{m}^{-3}$ & 0.00 & 0.01 & 0.02 & 0.03 & 0.13 & 0.03 \\
\hline Perylene & PER & $\mathrm{ng} \mathrm{m}^{-3}$ & 0.00 & 0.00 & 0.00 & 0.02 & 0.07 & 0.01 \\
\hline Indeno[1,2,3-cd]pyrene & IcdP & $\mathrm{ng} \mathrm{m}^{-3}$ & 0.02 & 0.02 & 0.04 & 0.05 & 0.09 & 0.04 \\
\hline Benzo[ghi]perylene & BghiP & $\mathrm{ng} \mathrm{m}^{-3}$ & 0.00 & 0.05 & 0.08 & 0.16 & 0.26 & 0.10 \\
\hline 9H-Fluoren-9-one & 9HFLUone & $\mathrm{ng} \mathrm{m}^{-3}$ & 0.29 & 0.48 & 0.59 & 0.76 & 2.24 & 0.75 \\
\hline 1,8-Naphthalic anhydride & NAP-AN & $\mathrm{ng} \mathrm{m}^{-3}$ & 0.12 & 0.18 & 0.27 & 0.95 & 4.71 & 0.92 \\
\hline Naphthoic acid & NAP-AC & $\mathrm{ng} \mathrm{m}^{-3}$ & 0.12 & 0.32 & 0.77 & 1.21 & 9.13 & 1.62 \\
\hline 9,10-Anthracenedione & $9,10-\mathrm{AN}$ & $\mathrm{ng} \mathrm{m}^{-3}$ & 0.29 & 0.48 & 0.59 & 0.76 & 2.24 & 0.75 \\
\hline
\end{tabular}



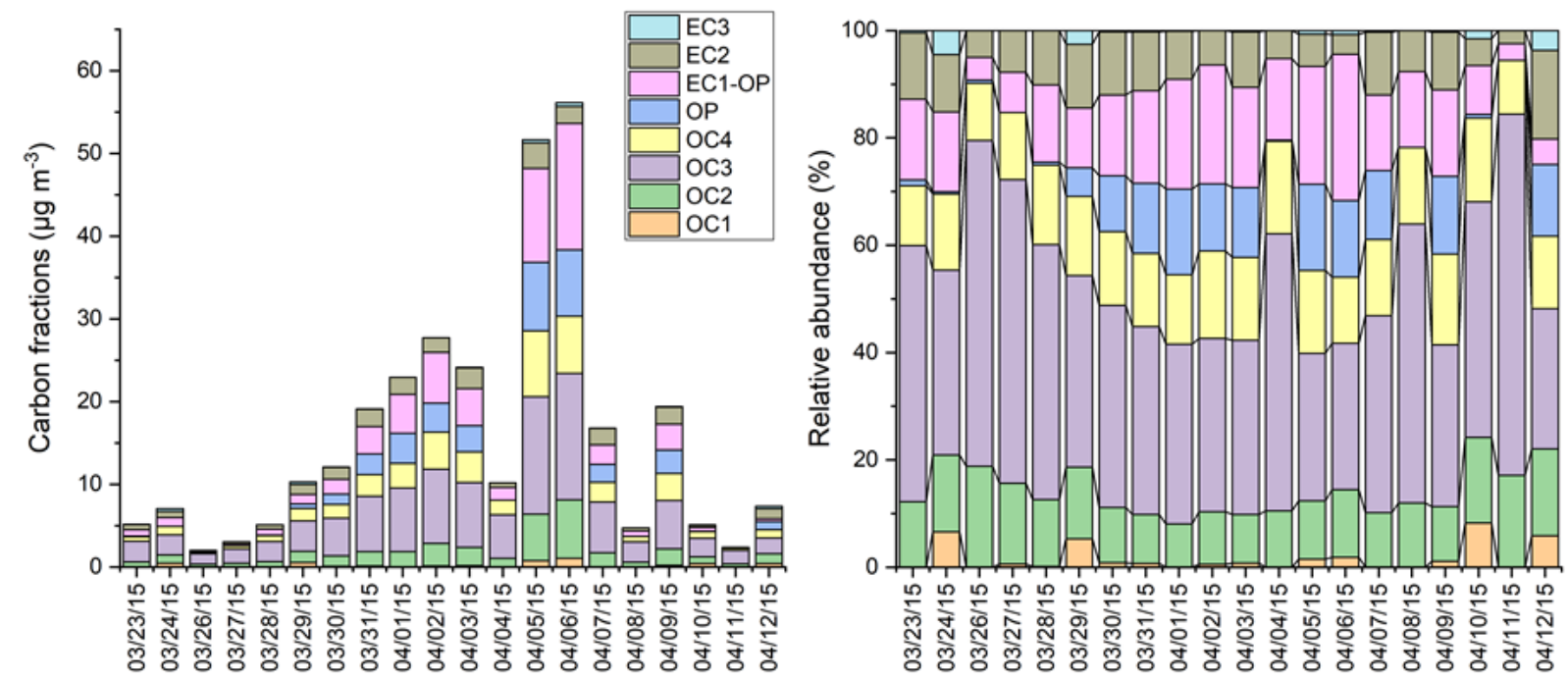

Figure S1. Time series of carbon fractions OC1, OC2, OC3, OC4, OP, EC1-OP, EC2 and EC3 in daily aerosol particle samples $(n=20)$ at PDI during the sampling campaign from 23rd March to $12^{\text {th }}$ April 2015. Left column provides concentrations of each carbon fraction $\left(\mu \mathrm{g} \mathrm{m}^{-3}\right)$, and right column provides relative mass fractions within carbonaceous fractions.
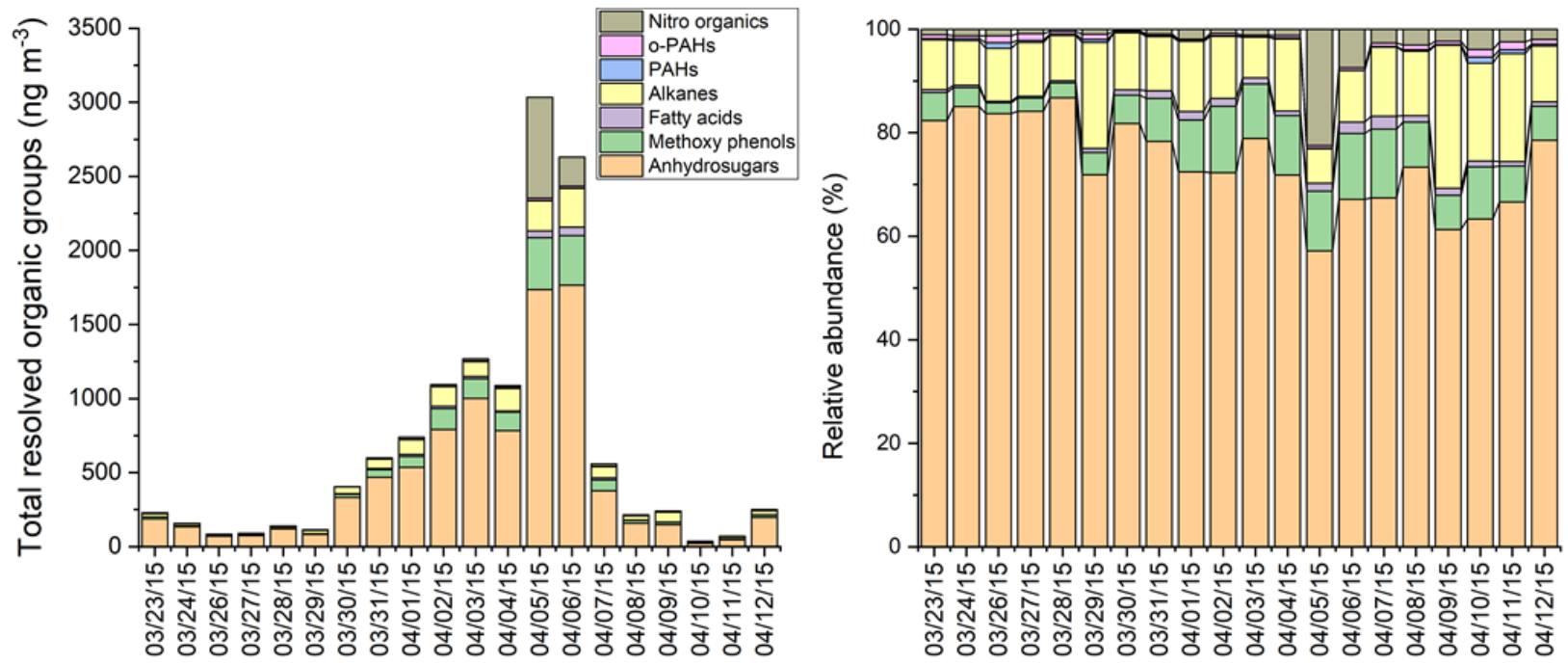

Figure S2. Time series of total resolved organic constituents in daily aerosol particle samples $(n=20)$ at PDI during the sampling campaign from $23^{\text {rd }}$ March to $12^{\text {th }}$ April 2015. Left column provides identified mass concentrations $\left(\mu \mathrm{g} \mathrm{m}^{-3}\right)$, and right column provides relative mass fractions within total class of compounds 


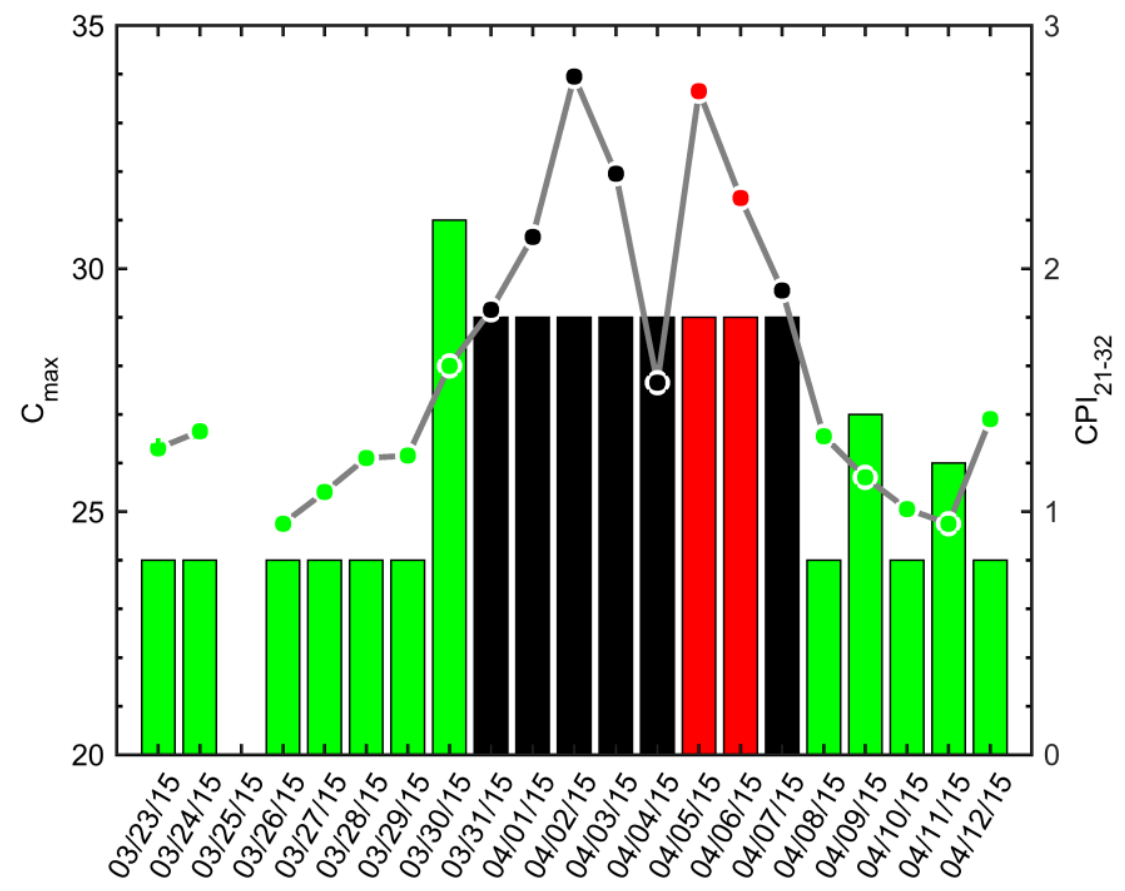

Figure S3. Carbon number of most abundant $\mathrm{n}$-alkane $\left(\mathrm{C}_{\max }\right.$; bars $)$ and Carbon Preference Index using n-alkanes from $\mathrm{C}_{21} \mathrm{H}_{44}$ to $\mathrm{C}_{32} \mathrm{H}_{66}\left(\mathrm{CPI}_{21-32}\right.$; circles).

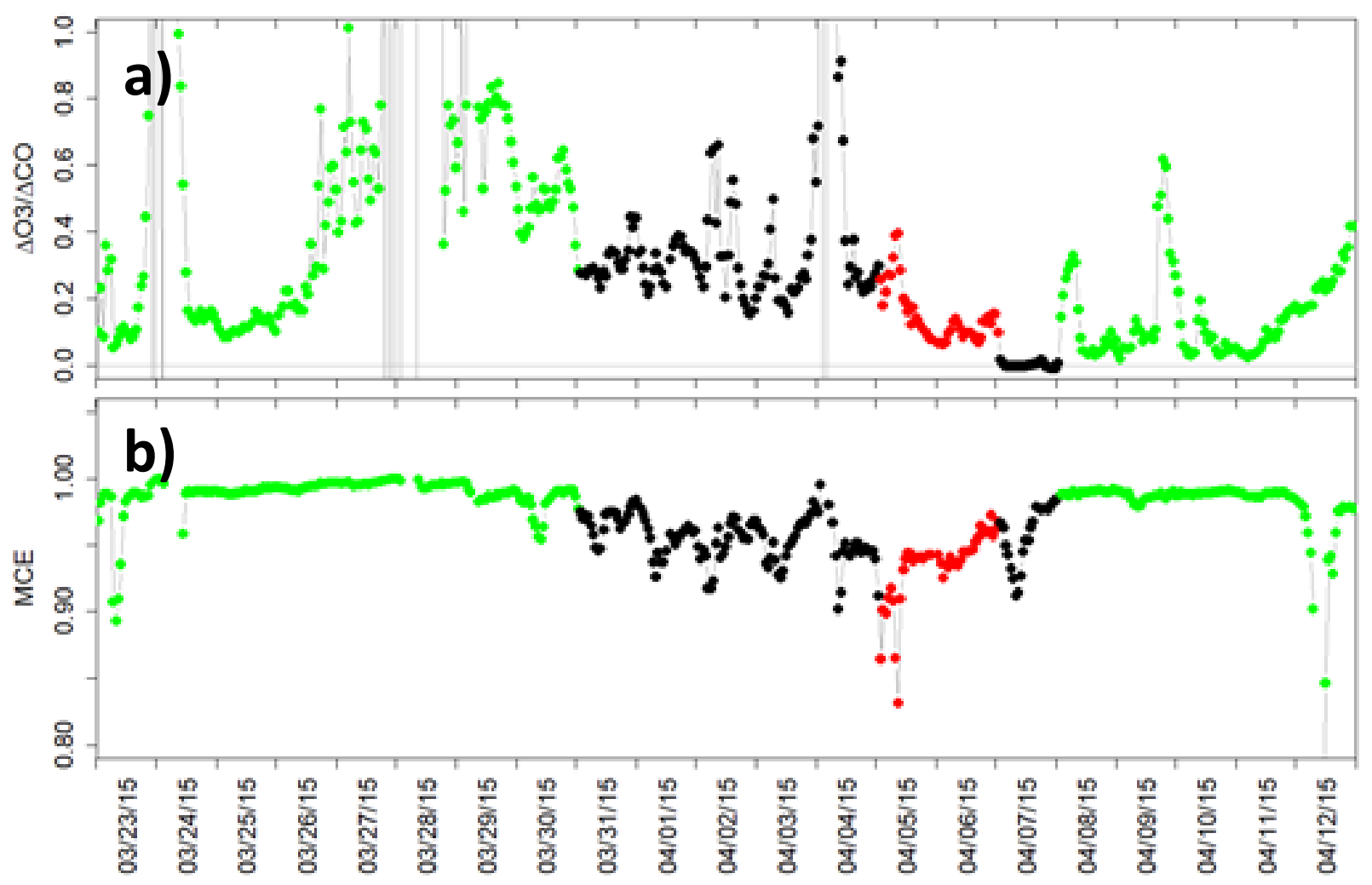

Figure S4. a) Hourly excess ratio between $\mathrm{O}_{3}$ and $\mathrm{CO}\left(\Delta \mathrm{O}_{3} /(\Delta \mathrm{CO})\right.$ ranged between $0.00-1.00$. b) Hourly modified combustion efficiency (MCE: $\Delta \mathrm{CO}_{2} /\left(\Delta \mathrm{CO}+\Delta \mathrm{CO}_{2}\right)$ ratios ranged between $0.80-$ 1.00 at PDI during the sampling campaign from $23^{\text {rd }}$ March to $12^{\text {th }}$ April 2015. Three colors, including green, black, and red follow the OA clustergram (see main text). 

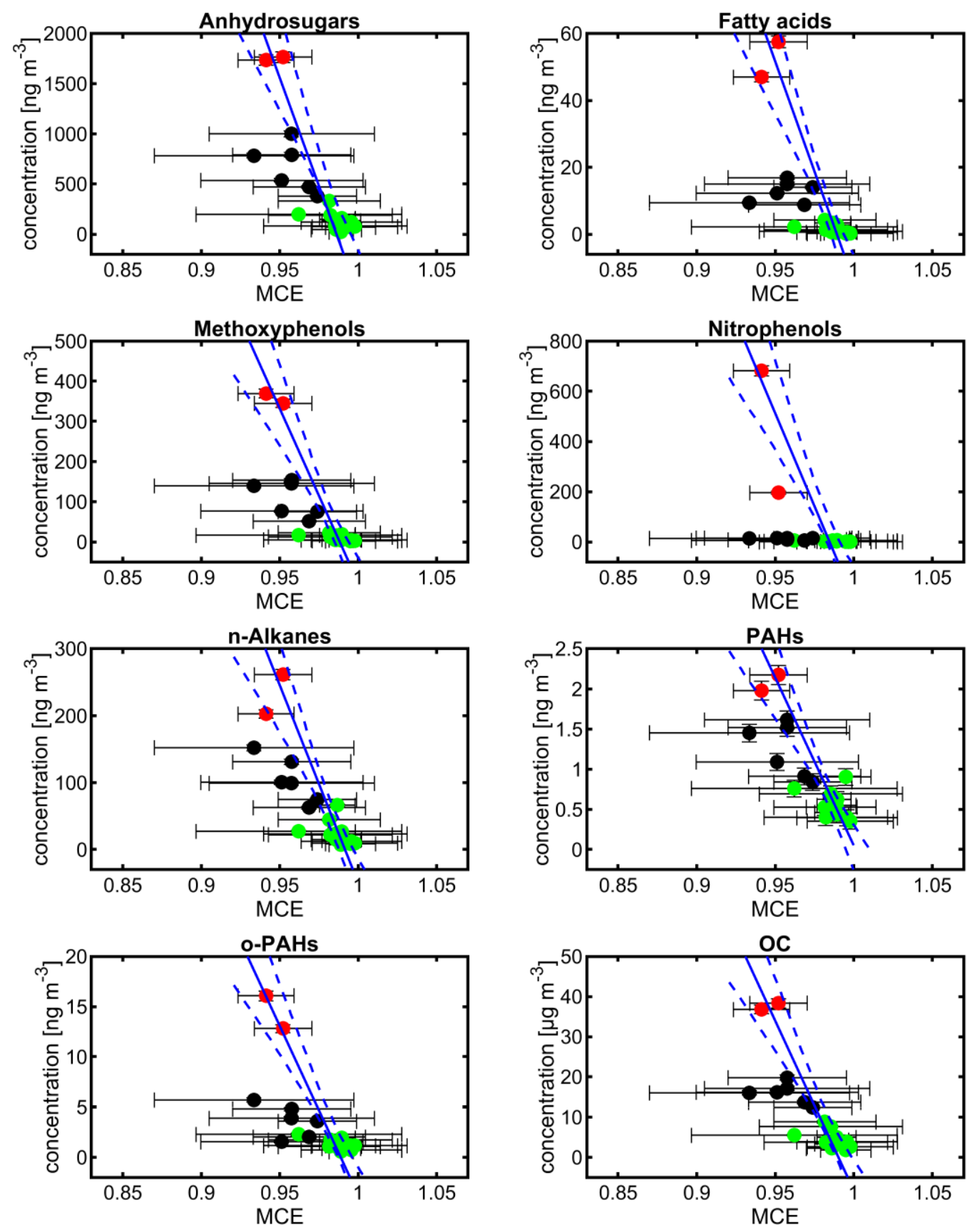

Figure S5. Relation between modified combustion efficiency (MCE) and various compound classes. Dots of green, black and red color correspond to the periods of low-BB, medium and high-BB, respectively. Black errorbars illustrate the uncertainty of each data point (please note that uncertainties for concentrations are too small to be visible in the figure). The blue lines refer to linear fits from Deming regression. Dashed lines denote the non-simultaneous prediction band of the fit function by means of one standard deviation. For most data points, concentration-related errorbars are too small to be visible. 
Table S1. Fit coefficients for Deming regression of concentration $=a \cdot M C E+b$ (Figure S4) and their uncertainty by means of one standard deviation from 500 Monte Carlo runs

\begin{tabular}{lll}
\hline Compound class & Slope $\mathrm{a}\left[\mathrm{ng} \mathrm{m}^{-3}\right]$ & Intercept $\mathrm{b}\left[\mathrm{ng} \mathrm{m}^{-3}\right]$ \\
\hline Anhydrosugars & $-4.30 \mathrm{e} 4 \pm 1.39 \mathrm{e} 4$ & $4.25 \mathrm{e} 4 \pm 1.36 \mathrm{e} 4$ \\
Fatty acids & $-1.31 \mathrm{e} 3 \pm 420$ & $1.30 \mathrm{e} 3 \pm 410$ \\
Methoxyphenols & $-8.68 \mathrm{e} 3 \pm 2.70 \mathrm{e} 3$ & $8.58 \mathrm{e} 3 \pm 2.67 \mathrm{e} 3$ \\
Nitrophenols & $-1.48 \mathrm{e} 4 \pm 5.04 \mathrm{e} 3$ & $1.46 \mathrm{e} 4 \pm 4.89 \mathrm{e} 3$ \\
n-Alkanes & $-5.90 \mathrm{e} 3 \pm 5.04 \mathrm{e} 3$ & $5.85 \mathrm{e} 3 \pm 4.89 \mathrm{e} 3$ \\
PAHs & $-41.8 \pm 14.0$ & $41.8 \pm 13.7$ \\
O-PAHs & $-337 \pm 101$ & $332 \pm 99$ \\
OC & $-844 \mathrm{e} 3 \pm 268 \mathrm{e} 3$ & $836 \mathrm{e} 3 \pm 263 \mathrm{e} 3$ \\
\hline
\end{tabular}

a)
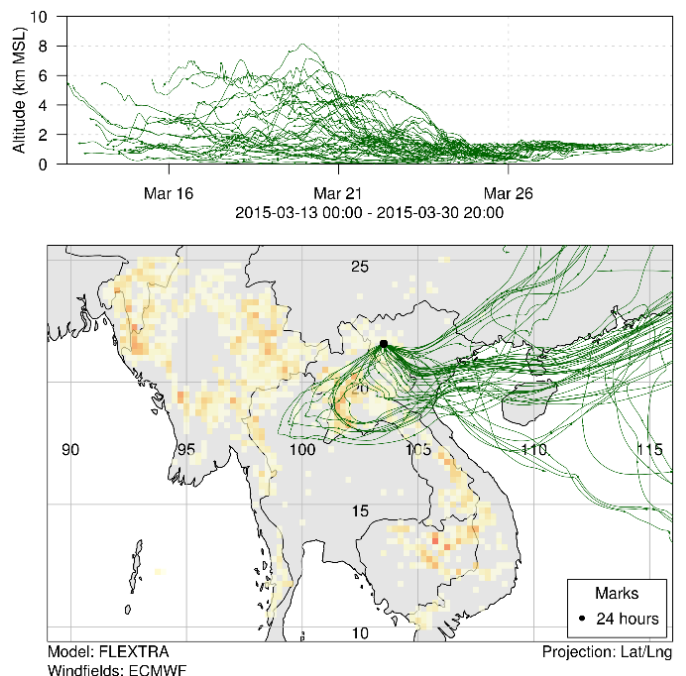

c)
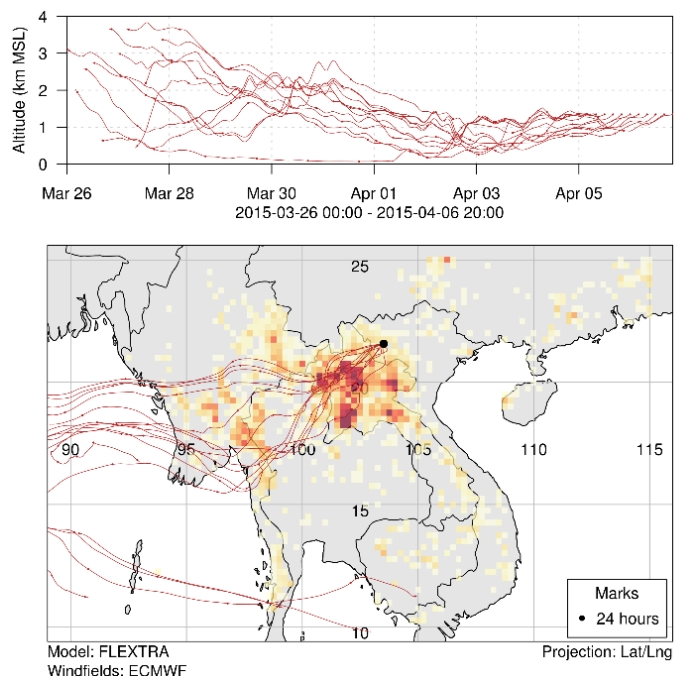

b)
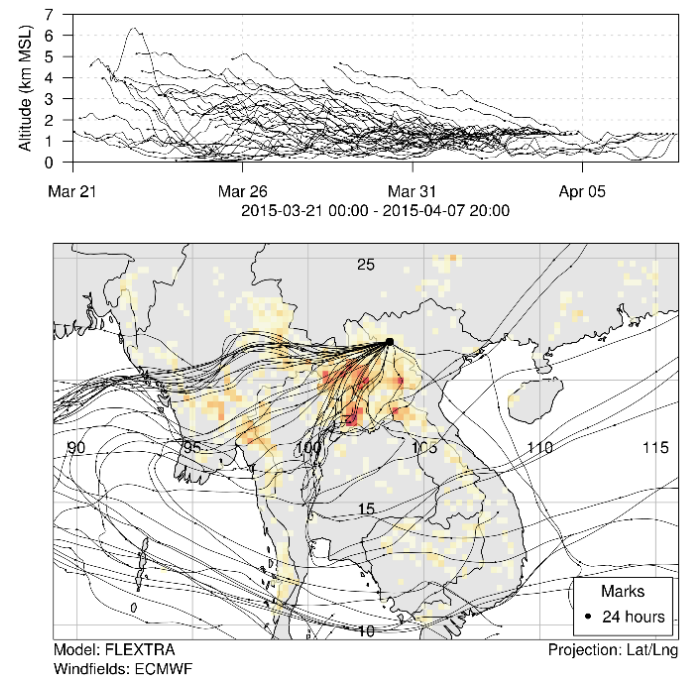

d)
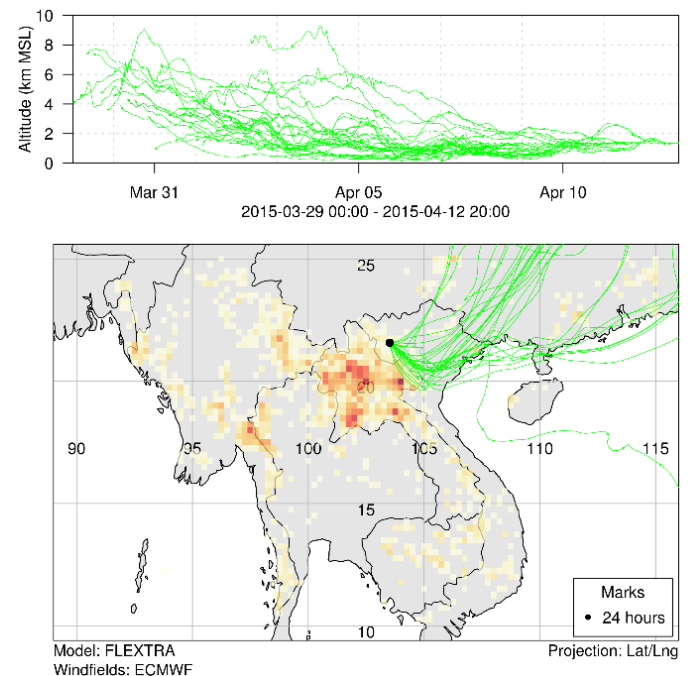

Figure S6. Ten-day backward trajectories arriving at PDI; the sub-periods were determined by the organic aerosol clustering (a) 23 - 30 March (n=8), b) 31 March- 4 April +7 April (n=6), c) 05 and 06 April (n=2), d) $8-12$ April $(n=6))$. The upper panels display the average height of the trajectories above sea level against time. The lower panel gives the average location of the trajectories overlaid on a map of MODIS fire count densities for the period five days before the beginning of each sub-period until the end of each sub-period (from low (bright yellow) to high (orange-red) fire intensities). 